\title{
A Diagram Calculus for Certain Canonical Bases
}

\author{
R.M. Green ${ }^{\star}$ \\ Mathematical Institute, Oxford University, 24-29 St. Giles', Oxford OX1 3LB, England. \\ E-mail: greenr@maths.ox.ac.uk
}

Received: 9 May 1996/ Accepted: 11 June 1996

\begin{abstract}
We introduce a certain cellular algebra $Q(n, r)$ which is a quotient of the $q$-Schur algebra $S_{q}(n, r)$. This is naturally equipped with a canonical basis which is compatible with Lusztig's canonical bases for certain modules for the quantized enveloping algebra $U\left(s l_{n}\right)$. We describe a diagram calculus for $Q(n, r)$ which makes calculations involving the corresponding canonical bases easy to understand.
\end{abstract}

\section{Introduction}

The $q$-Schur algebra $S_{q}(n, r)$, which was introduced by Dipper and James in [2], is a certain finite-dimensional associative algebra over the ring of Laurent polynomials $\mathcal{A}=\mathbb{Z}\left[v, v^{-1}\right]$. Du [3] has described a certain natural free $\mathcal{A}$-basis $\left\{\theta_{\lambda, \mu}^{w}\right\}$ for the $q$ Schur algebra, which we shall refer to as the canonical basis. It is known [8, Corollary 4.7] that the structure constants with respect to this basis have interpretations in the framework of perverse sheaves and intersection cohomology. It is also known (see for example [5, Theorem 4.6]) that the basis $\left\{\theta_{\lambda, \mu}^{w}\right\}$ is very closely related to Lusztig's canonical bases for modules quantized enveloping algebras, which were defined in [9]. These bases have turned out to be very important in representation theory.

In Sect. 2 we define and study a certain quotient $Q(n, r)$ of $S_{q}(n, r)$ which inherits a basis from the basis $\left\{\theta_{\lambda, \mu}^{w}\right\}$ of $S_{q}(n, r)$. This quotient $Q(n, r)$ is closely related to the Temperley-Lieb algebra $T^{H} L_{r}$ of type $A$. Using the main result of [6], we show that the multiplication in $Q(n, r)$ can be easily described in terms of a diagram calculus which is an extension of the $r$-diagram calculus for the Temperley-Lieb algebra (described for example in [12, Sect. 1]). Furthermore, the diagrams can be interpreted as canonical basis elements of $S_{q}(n, r)$.

In Sect. 3 we describe the cellular structure of $Q(n, r)$ (in the sense of [7]) and classify the absolutely irreducible modules for $Q(n, r)$ over a field.

\footnotetext{
* The author was supported in part by an E.P.S.R.C. postdoctoral research assistantship.
} 
In Sect. 4 we use the homomorphism $\pi_{r}$ from an $\mathcal{A}$-form $U=U_{\mathcal{A}}\left(s l_{n}\right)$ of the quantized enveloping algebra $U\left(s l_{n}\right)$ to $S_{q}(n, r)$ (as in [4]) to describe a left action of $U$ on $Q(n, r)$ which gives rise to the action of $U$ on the canonical basis for its irreducible highest weight modules over a field.

Since the results of this paper tie together Temperley-Lieb algebras (which were defined in [11] and were motivated by the Potts model in statistical mechanics) and the quantized enveloping algebras of Drinfel'd and Jimbo (which arose from the study of the quantum inverse scattering method), one would expect there to be some physical interpretation of these results.

\section{The algebra $Q(n, r)$}

It turns out that the result [6, Theorem 3.8.2], which shows that the product of two "canonical" basis elements in $T L_{r}$ is a scalar multiple of another one, is a manifestation of a more general, but equally elegant, result which applies to the representation theory of $q$-Schur algebras and quantized enveloping algebras.

Following [3, Sect. 2], we define $\Lambda=\Lambda(n, r)$ to be the set of compositions $\lambda \vDash r$ of $r$ into $n$ pieces. We shall assume until further notice that $n \geq r$, so there exists an element $\omega \in \Lambda$ given by $\omega=(\underbrace{1,1, \ldots, 1}_{r}, 0, \ldots 0)$. To a composition $\lambda$ of $r$ we associate in the natural way a parabolic subgroup $W_{\lambda}$ of the symmetric group $\mathcal{S}_{r}$.

Let $\lambda$ and $\mu$ be arbitrary elements of $\Lambda$. To each Kazhdan-Lusztig basis element $B_{w}$ of $\mathcal{H}$, where $w$ is the double coset representative of maximal length for the double coset $W_{\lambda} w W_{\mu}$, we define an element $\theta_{\lambda, \mu}^{w}$. The set of all such $\theta_{\lambda, \mu}^{w}$ spans an associative algebra called the $q$-Schur algebra and denoted by $S_{q}(n, r)$.

Let us denote by $g_{a, b, c}$ the coefficient of $B_{c}$ in $B_{a} B_{b}$. Then the multiplication in the $q$-Schur algebra is given by

$$
\theta_{\lambda \mu}^{a} \theta_{\nu \rho}^{b}=\delta_{\mu, \nu}\left(\sum_{c} f_{a, b, c} \theta_{\lambda, \rho}^{c}\right)
$$

where

$$
h_{\mu}=v^{-\ell\left(w_{0, \mu}\right)} \sum_{w \in W_{\mu}} v^{2 \ell(w)}
$$

( $w_{0, \mu}$ being the longest element of $W_{\mu}$ ) and

$$
h_{\mu} f_{a, b, c}=g_{a, b, c} .
$$

The Hecke algebra $\mathcal{H}=\mathcal{H}\left(A_{r-1}\right)$ corresponding to the Coxeter group of type $A_{r-1}$ may naturally be identified with the subalgebra of $S_{q}(n, r)$ spanned by the basis elements $\theta_{\omega, \omega}^{w}$ (corresponding to the basis elements $B_{w}$ ).

The Temperley-Lieb algebra $T L_{r}$ of type $A$ is the quotient of $\mathcal{H}$ by the ideal $J$ spanned by all basis elements $B_{w}$ where $w$ corresponds under the Robinson-Schensted map to a pair of tableaux with at least 3 columns ([6, Proposition 3.1.1]). (A good reference for the Robinson-Schensted correspondence is [10].) Following [6], we denote the nonzero images in $\mathcal{H} / J$ of elements $B_{w} \in \mathcal{H}$ by $F_{w}$. It was shown in [6, Theorem 3.8.2] that the basis of elements $F_{w}$ coincides with the basis of $r$-diagrams as given in $[12$, Sect. 1]. From now on we make this identification implicitly. 
Definition 2.1.1. Let $n \geq r$. We denote by $Q(n, r)$ the quotient of $S_{q}(n, r)$ by the ideal $I$ spanned by elements $\theta_{\lambda, \mu}^{w}$, where $w$ corresponds to a tableau with at least three columns.

Note that it is clear from the definitions that the given elements span an ideal, because the corresponding elements of $\mathcal{H}$ do (see [6, Proposition 3.1.1]). Thus $Q(n, r)$ inherits a canonical basis from the $\theta$-basis of $S_{q}(n, r)$. We write the elements of this as $\zeta_{\lambda, \mu}^{x}$.

Lemma 2.1.2. Let $\zeta_{\lambda, \mu}^{x}$ be a canonical basis element of $Q(n, r)$. Then $W_{\lambda}$ and $W_{\mu}$ are each generated by sets of commuting simple reflections, and $x$ corresponds (via the Robinson-Schensted algorithm) to a pair of tableaux with one or two columns.

Proof. We recall that a semistandard tableau is one in which the entries increase weakly along rows, and strictly down the columns. It is known from [5, Sect. 3] that the basis of elements $\theta_{\lambda, \mu}^{w}$ is naturally parametrised by pairs of semistandard tableaux of the same shape as the tableaux corresponding to $w$; furthermore, the content of the left tableau is $\lambda$ and the content of the right tableau is $\mu$. It is clear from the definition of $Q(n, r)$ that $x$ corresponds to a pair of tableaux with one or two columns. The condition of semistandardness now forces all the components of the compositions $\lambda$ and $\mu$ to have size 0,1 or 2 . The associated Weyl groups $W_{\lambda}$ and $W_{\mu}$ are determined by the components of size 2, which correspond to commuting simple reflections which generate the groups.

It is convenient to represent $\zeta_{\lambda, \mu}^{w}$ as a diagram, thus generalising the $r$-diagrams for Temperley-Lieb algebras.

Definition 2.1.3. A generalised r-diagram consists of a pair $\langle\lambda, \mu\rangle$ of elements of $\Lambda(n, r)$ and two rows of $r$ nodes together with $r$ edges linking pairs of nodes. The elements $\lambda$ and $\mu$ correspond to compositions of $r$ into at most $n$ pieces, and the pieces must be of size 2, 1 or 0 . Each node is the endpoint of exactly one edge, and the edges must not intersect. Edges are of three types: vertical, which link nodes in different rows; flat, which link adjacent nodes in the same row; and horizontal, which link (possibly adjacent) nodes in the same row. The diagram has a flat edge linking points $i$ and $i+1$ in the top row if and only if $(i, i+1) \in W_{\lambda}$, and a flat edge linking points $i$ and $i+1$ in the bottom row if and only if $(i, i+1) \in W_{\mu}$.

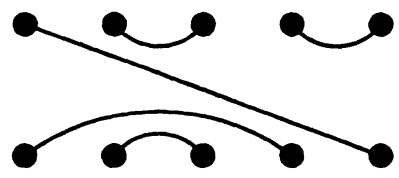

Fig. 1. An $r$-diagram $(r=5)$

Figure 2 gives an example of a generalised $r$-diagram corresponding to the pair $\langle\lambda, \mu\rangle$, where $W_{\lambda}$ is generated by the transposition $(4,5)$ and $W_{\mu}$ is generated by the transposition $(2,3)$. (Note that $\lambda$ and $\mu$ are not defined by this condition.) This corresponds to the element $\zeta_{\lambda, \mu}^{w}$, where $F_{w}$ corresponds to the diagram in Fig. 1 (which is the same as this figure with the labels erased and the flat edges, denoted by straight lines, replaced by horizontal ones).

Two generalised $r$-diagrams $A$ and $B$ multiply in the following way. If $A$ has pair $\langle\lambda, \mu\rangle$ and $B$ has pair $\langle\nu, \rho\rangle$ then the product $A B$ is zero unless $\mu=\nu$. In this case 


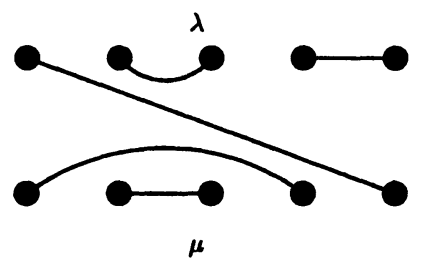

Fig. 2. An generalized $r$-diagram

we place the diagram for $A$ above $B$, identify the lower row of $A$ with the upper row of $B$, and remove all the nodes in the new combined row. This process results in a new diagram, $C$, and produces some loops which are of two types: those which are constructed entirely of flat edges and those which are constructed entirely of horizontal, but not flat, edges. Suppose there are $x$ loops which do not consist entirely of flat edges. Then $A B=[2]^{x} C$, where $C$ is labelled by the pair $\langle\lambda, \rho\rangle$. (Note that the "flat loops" have effectively been ignored.)

The point of introducing generalised $r$-diagrams is that the multiplication in $Q(n, r)$ is easy to understand in terms of them.

Theorem 2.1.4. The $\mathcal{A}$-linear map sending $\zeta_{\lambda, \mu}^{w}$ to the generalised $r$-diagram corresponding to the $r$-diagram for $w$ and the pair $\langle\lambda, \mu\rangle$ is a monomorphism of $\mathcal{A}$-algebras.

Proof. First we note that $w$ is the longest element in the double coset $W_{\lambda} w W_{\mu}$ if and only if $s w<w$ for each $s \in W_{\lambda}$ and $w s<w$ for each $s \in W_{\mu}$. One sees easily that this is equivalent to the $r$-diagram for $F_{w}$ having points $i$ and $i+1$ joined in the top row when $(i, i+1) \in W_{\lambda}$, and having points $i$ and $i+1$ joined in the bottom row when $(i, i+1) \in W_{\mu}$. So different basis elements of $Q(n, r)$ do indeed correspond to different generalised $\boldsymbol{r}$-diagrams.

It remains to show that the multiplication is correct. This comes from comparison with the multiplication of the $F$-basis (governing the Temperley-Lieb algebra), the $B$-basis of the Hecke algebra and the $\theta$-basis of $S_{q}(n, r)$. One needs to ensure that the product is zero when the weights are incompatible (which is true), and that when the weights are compatible, the product is $1 / h_{\mu}$ times the corresponding product in the Temperley-Lieb algebra or Hecke algebra. Thanks to Lemma 2.1.2, $h_{\mu}$ is equal to [2] where $x$ is the number of commuting reflections generating $W_{\mu}$, or, equivalently, the number of "flat loops" formed in the multiplication of the diagrams $A$ and $B$. The proof now follows.

\section{The Cellular Structure of $Q(n, r)$}

The next aim is to show that the algebra $Q(n, r)$ is a cellular algebra in the sense of [7].

3.1. Generalised Parenthesis Diagrams. In this section we generalise the parenthesis diagrams which appeared in [12, Sect. 2]. These were certain diagrams in correspondence with standard tableaux with at most two rows (and hence in correspondence with standard tableaux with at most two columns). We wish to find a set of diagrams which are in correspondence with semistandard tableaux with at most two columns. 
Definition 3.1.1. A generalised $(r, p)$-parenthesis diagram consists of:

1. a label, which is an element $\lambda \in \Lambda(n, r)$ satisfying $\lambda_{i} \leq 2$ for all $i$;

2. a graph, which is a row of $r$ points on the $x$-axis together with $p$ non-intersecting edges which lie in the lower half plane, each of which joins precisely two of the points. The edges are of two types: horizontal and flat. A flat edge occurs between points $i$ and $j$ if and only if $j=i+1$ and $(i, i+1)$ lies in the Weyl group $W_{\lambda}$. If points $a, b, c$ satisfy $a<b<c$ and $a$ is connected to $c$, then $b$ is required to be connected to some other point.

Example 3.1.2. Figure 3 shows a generalised (5, 2)-diagram whose corresponding Weyl group $W_{\lambda}$ is generated by the simple transposition $(3,4)$.

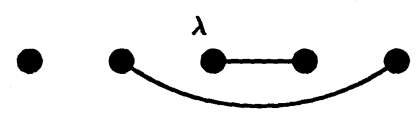

Fig. 3. A generalized $(5,2)$-parenthesis diagram

Remark 3.1.3. By placing an $(r, p)$-parenthesis diagram, $a$, on top of an inverted $(r, p)$ parenthesis diagram $b$, and connecting the isolated points with non-intersecting vertical edges, one obtains a generalised $r$-diagram with $p$ vertical edges. Every generalised $r$-diagram arises uniquely in this way. We denote the resulting generalised $r$-diagram by $|a\rangle\langle b|$. (Recall that the product $\left|a_{1}\right\rangle\left\langle b_{1} \| a_{2}\right\rangle\left\langle b_{2}\right|$ is some power of [2] times another element $\left|a_{3}\right\rangle\left\langle b_{3}\right|$.)

3.2. Connections with semistandard tableaux. We now show that generalised $(r, p)$ parenthesis diagrams are in natural correspondence with semistandard tableaux with at most two columns and $r$ boxes in total, $p$ of which are in the second column.

Definition 3.2.1. Let $\lambda \in \Lambda(n, r)$. We define the ordered $r$-tuple $\ell(\lambda)$ of integers $\left(\ell(\lambda)_{1}, \ldots, \ell(\lambda)_{r}\right)$ to be the unique weakly increasing sequence where the integer $s$ occurs $\lambda_{\text {s }}$ times.

Definition 3.2.2. We may identify an $(r, p)$-parenthesis diagram $D$ with graph $g$ and label $\lambda$ with a pair consisting of a graph $g$ and a function $\rho: \mathbf{r} \rightarrow \mathbf{n}$ taking $i$ to $\ell(\lambda)_{i}$. We call $\rho(i)$ the value of the $i^{\text {th }}$ point in the diagram $D$.

Note that points $i$ and $i+1$ have the same value if and only if they are connected by a flat edge.

Definition 3.2.3. We say point $i$ of the diagram $D$ is of type 2 if it is connected by a (flat or horizontal) edge to a point to its left. Otherwise we say $i$ is of type 1, which means that it is isolated or connected by a (flat or horizontal) edge to a point to its right.

Proposition 3.2.4. Let $D$ be a generalised $(r, p)$-parenthesis diagram. Let $T_{D}$ be the unique column-standard tableau with two columns and $r$ boxes in total, $p$ of which are in the second column, satisfying the conditions that the $a^{\text {th }}$ column of $T_{D}$ consists of the family $\rho(i)$ as $i$ ranges over points of $D$ of type a. Then $T_{D}$ is semistandard and every semistandard tableau with at most two columns arises from a unique generalised $(r, p)$-parenthesis diagram in this way. 
Example 3.2.5. Let $n=6, r=5, \lambda=(1,0,1,2,0,1)$. Then $\ell(\lambda)=(1,3,4,4,6)$ and $W_{\lambda}=\langle(3,4)\rangle$. (Recall that $W_{\lambda}$ is the stabiliser of $\ell(\lambda)$ and not of $\lambda$.) Now consider the diagram $D$ shown in Fig. 3. Points 4 and 5 are of type 2, and the others are of type 1. This corresponds to the semistandard tableau $T_{D}$ given by

\begin{tabular}{|l|l|}
\hline 1 & 4 \\
\hline 3 & 6 \\
\hline 4 & \multicolumn{1}{|l}{} \\
\cline { 1 - 1 } &
\end{tabular}

Proof of Proposition 3.2.4. Let $D$ be a generalised $(r, p)$-parenthesis diagram. After replacing the flat edges with normal horizontal edges, this corresponds to a parenthesis diagram in the sense of [12] together with a function $\rho$ as in Definition 3.2.2. Using Westbury's correspondence [12, Sect. 2] between parenthesis diagrams and standard tableaux with at most two columns and $p$ boxes in the second column, we obtain a standard tableau together with the function $\rho$. We now relabel the entries of the tableau by replacing $i$ with $\rho(i)$. We claim this is semistandard. The fact that the rows are weakly increasing follows from the monotonicity of $\rho$. The only possible problem is that we have a repeated entry in one of the columns. This cannot happen because if there are two points with the same value in the diagram $D$, one must be of type 1 and the other of type 2.

The inverse of the above correspondence can be described as follows. Start with a semistandard tableau $T$. There is a unique standard tableau $T^{\prime}$ and a function $\rho: \mathbf{r} \rightarrow \mathbf{n}$ such that (a) $T$ is the relabelling of $T^{\prime}$ by $\rho$ (as above) and (b) if $T$ has identical entries in position $a$ of column 1 and position $b$ of column 2, then the entry in position $a$ of column 1 of $T^{\prime}$ is one less than the entry in position $b$ of column 2 of $T^{\prime}$. Referring to Example 3.2.5, we would find that $T^{\prime}$ was equal to

\begin{tabular}{|l|l|}
\hline 1 & 4 \\
\hline 2 & 5 \\
\hline 3 & \multicolumn{1}{|l}{} \\
\cline { 1 - 1 } &
\end{tabular}

rather than

\begin{tabular}{|l|l|}
\hline 1 & 3 \\
\hline 2 & 5 \\
\hline 4 & \multicolumn{1}{|l}{} \\
\cline { 1 - 1 } &
\end{tabular}

We then form the parenthesis diagram corresponding to $T^{\prime}$ as described by Westbury. If points $i$ and $i+1$ are connected whenever $(i, i+1) \in W_{\lambda}$ (where $\lambda$ is such that $\ell(\lambda)$ corresponds to $\rho$ ), then we are done since we can flatten all the relevant edges. This is indeed the case; if $(i, i+1) \in W_{\lambda}$ then $\rho(i)=\rho(i+1)$ which means that point $i+1$ is the right endpoint of an edge and point $i$ is the left endpoint of an edge. It follows by the non-intersection condition that they must therefore be joined. This completes the proof.

Remark. Notice that the set of basis elements of $Q(n, r)$ corresponding to pairs of standard rather than semistandard tableaux is canonically isomorphic to the TemperleyLieb algebra $T L_{r}$ of type $A$. 
Definition 3.3.1. (Graham, Lehrer) Let $R$ be a commutative ring with identity. $A$ cellular algebra over $R$ is an associative unital algebra, $A$, together with a cell datum $(\Lambda, M, C, *)$ where

1. $\Lambda$ is a poset. For each $\lambda \in \Lambda, M(\lambda)$ is a finite set (the set of tableaux of type $\lambda$ ) such that

$$
C: \coprod_{\lambda \in \Lambda}(M(\lambda) \times M(\lambda)) \rightarrow A
$$

is injective with image an $R$-basis of $A$.

2. If $\lambda \in \Lambda$ and $S, T \in M(\lambda)$, we write $C(S, T)=C_{S, T}^{\lambda} \in A$. Then $*$ is an R-linear involutory anti-automorphism of $A$ such that $\left(C_{S, T}^{\lambda}\right)^{*}=C_{T, S}^{\lambda}$.

3. If $\lambda \in \Lambda$ and $S, T \in M(\lambda)$, then for all $a \in A$ we have

$$
a . C_{S, T}^{\lambda} \equiv \sum_{S^{\prime} \in M(\lambda)} r_{a}\left(S^{\prime}, S\right) C_{S^{\prime}, T}^{\lambda} \bmod A(<\lambda)
$$

where $r_{a}\left(S^{\prime}, S\right) \in R$ is independent of $T$ and $A(<\lambda)$ is the $R$-submodule of $A$ generated by the set

$$
\left\{C_{S^{\prime \prime}, T^{\prime \prime}}^{\mu}: \mu<\lambda, S^{\prime \prime} \in M(\mu), T^{\prime \prime} \in M(\mu)\right\}
$$

Proposition 3.3.2. Let $R$ be the subring $\mathbb{Z}\left[t, t^{-1}\right]$ of $\mathcal{A}$, where $t=[2]$. Let $\Lambda^{\prime}$ be the set of integers from 0 to $\left\lfloor\frac{r}{2}\right\rfloor$ inclusive, ordered by the reverse of the usual order on the integers. For $p \in \Lambda^{\prime}$, let $M(p)$ be the set of generalised $(r, p)$-parenthesis diagrams. Let $*$ be the anti-automorphism of $Q(n, r)$ corresponding to top-bottom inversion of the diagrams. Let $C$ be the map taking two generalised $(r, p)$ diagrams with the same value of $p$ to a generalised $r$-diagram as explained in Remark 3.1.3.

Then, working over $R, Q(n, r)$ is a cellular algebra with cell datum $\left(\Lambda^{\prime}, M, C, *\right)$.

Proof. The fact that all the axioms are satisfied follows easily from the definition of $Q(n, r)$.

Corollary 3.3.3. The algebra $Q(n, r)$ is quasi-hereditary.

Proof. Note that the element $\lambda=\lambda_{k}$ in $\Lambda(n, r)$ given by

$$
(\underbrace{2, \ldots, 2}_{k}, \underbrace{1, \ldots, 1}_{r-2 k}, 0, \ldots 0)
$$

gives rise to the basis element $\zeta_{\lambda, \lambda}^{w^{+}}$of $Q(n, r)$, where $w^{+}$is the longest element in $W_{\lambda}$. This is idempotent and corresponds to an element in the cell labelled by $k \in \Lambda^{\prime}$, and this phenomenon occurs for all $k$. It follows from [7, Remark 3.10] that $Q(n, r)$ is quasi-hereditary. 


\section{Connections with Lusztig's Canonical Bases}

In this section we take the base ring of $Q(n, r)$ to be a field, $K$, containing $\mathbb{Z}[t]$, where $t=[2]$, unless otherwise stated.

4.1. The cell modules for $Q(n, r)$. We now describe the cell representations (in the sense of [7, Sect. 2]) for $Q(n, r)$ in terms of the calculus of generalised $(r, p)$-diagrams.

Associated with the $p^{\text {th }}$ cell module we have a bilinear form $\phi_{p}(x, y)$ as follows.

Definition 4.1.1. Let $x$ and $y$ be generalised $(r, p)$-parenthesis diagrams. Then we define a bilinear form $\phi_{p}$ on $W(p)$ by the condition that $\phi_{p}(x, y)$ is the coefficient of $|a\rangle\langle b|$ in $|a\rangle\langle x|| y\rangle\langle b|$. We will also write $\langle x, y\rangle$ for $\phi_{p}(x, y)$.

Remark. Such a bilinear form $\phi$ is a standard feature of cellular algebras: see [7, Definition 2.3]. It follows from that theory that $\phi_{p}(x, y)$ does not depend on $a$ or $b$.

Definition 4.1.2. Let $p \in \Lambda^{\prime}$. We define the $p^{\text {th }}$ cell module $W(p)$ to be the span of all the generalised $(r, p)$-parenthesis diagrams with left $Q(n, r)$-action given by

$$
|a\rangle\langle b| . c=\phi_{p}(b, c) a .
$$

We define the $p^{\text {th }}$ cell representation to be that afforded by the $p^{\text {th }}$ cell module.

Remark. The fact that this is well-defined follows from Axiom 3 of Definition 3.3.1 and Proposition 3.3.2.

Definition 4.1.3. We write $\operatorname{rad}(p)$ for the subspace of the $p^{\text {th }}$ cell module given by

$$
\left\{x \in W(p): \phi_{p}(x, y)=0 \text { for all } y \in W_{p}\right\} .
$$

Remark. The theory of cellular algebras shows that $\operatorname{rad}(p)$ is a submodule of $W(p)$.

Proposition 4.1.4. The set of all $W(p) / \operatorname{rad}(p)$, where $p \in \Lambda^{\prime}$ is a set of (nontrivial) pairwise nonisomorphic absolutely irreducible modules for $Q(n, r)$ over a field.

Proof. Recall from the proof of Corollary 3.3.3 that for each $k \in \Lambda^{\prime}$ there exists an idempotent $\zeta_{\lambda, \lambda}^{\omega^{+}} \in Q(n, r)$ which we shall denote by $\left|\ell_{k}\right\rangle\left\langle\ell_{k}\right|$, where $\ell_{k}$ is a certain generalised $(r, k)$-diagram. (In fact, $\ell(k)$ is the diagram with label $\lambda$ whose graph has $k$ flat edges on the left and no other edges.) Since this element is idempotent it follows that $\left\langle\ell_{k}, \ell_{k}\right\rangle$ is nonzero, and thus that $\phi_{k}$ is nonzero. The proof now follows from [7, Theorem 3.4].

4.2. Compatibility with Lusztig's Canonical Basis. The next aim is to show that the basis of generalised $(r, p)$-diagrams for $W(p)$ is, under suitable identifications, the same as Lusztig's canonical basis.

Let us now assume that the field $K$ is of characteristic 0 and contains the indeterminate $v$, and hence the field $\mathbb{Q}(v)$. We regard $W(p)$ as a module for the $q$-Schur algebra $S_{q}(n, r)^{K}$ over the field $K$ in the obvious way (recall that $Q(n, r)$ was defined as a quotient of $S_{q}(n, r)$ ). 
Lemma 4.2.1. Let $p \in \Lambda^{\prime}$. Let $\mu \in \Lambda(n, r)$ be the weight

$$
(\underbrace{2, \ldots, 2}_{p}, \underbrace{1, \ldots, 1}_{r-2 p}, 0, \ldots 0) .
$$

Then in the notation of [5], $W(p)$ is canonically isomorphic to $V(\mu)$.

Proof. So long as we keep $v$ as an indeterminate, [5, Sect. 1.3] tells us that the $q$-Weyl module $V(\mu)$ is an irreducible module for $S_{q}(n, r)^{K}$ of highest weight $\mu$. The weights of the modules $W(p)$ are the elements of $\Lambda(n, r)$ associated to the diagram basis elements. They are also the contents of semistandard tableaux of shape $\mu$, which means that the most dominant weight is $\mu$ and the unique basis element of $W(p)$ of this weight is $\ell_{p}$ (in the notation of the proof of Proposition 4.1.4). Proposition 4.1.4 now implies that $V(\mu)$ is a quotient of $W(p)$, but the semistandard basis theorem for $q$-Weyl modules (see e.g. [5, Theorem 1.5]) implies that the dimension of both modules is the number of semistandard tableaux of shape $\mu$. Thus we have equality, as required.

Corollary 4.2.2. The module $W(p)$ is irreducible.

Proof. The dimension argument above forces $\operatorname{rad}(p)=0$, and the conclusion follows from Proposition 4.1.4.

Theorem 4.2.3. The basis of generalised diagrams for $W(p)$ agrees with Lusztig's canonical basis for $V(\mu)$.

Proof. It is explained in [5, Theorem 4.6] that Lusztig's canonical basis for $V(\mu)$ (regarded as a module for $U\left(s l_{n}\right)$ ) is the same as Du's canonical basis for $V(\mu)$ (regarded as a module for $S_{q}(n, r)$ ).

Let $v_{0}$ be a highest weight vector in $V(\mu)$ (which defines $v_{0}$ up to nonzero scalar multiples). By Lemma 4.2.1, we may choose $v_{0}$ to be the diagram $\ell_{p}$. Then Du's canonical basis for $V(\mu)$ is given (see [5, Theorem 4.6]) by the elements

$$
\left\{\theta_{\lambda, \nu}^{w^{+}} v_{0}: \theta_{\lambda, \nu}^{w^{+}} v_{0} \neq 0\right\} \text {. }
$$

It follows that the required elements $\theta$ are those that map to elements $\zeta \in Q(n, r)$, where $\zeta$ is of form $|x\rangle\left\langle\ell_{p}\right|$ for a generalised $(r, p)$-parenthesis diagram $x$. Thus the generalised $(r, p)$-parenthesis diagrams coincide with the canonical basis.

4.3. Action of the generators of $U$ on the diagrams. An important part of the representation theory of quantized enveloping algebras is their so-called highest weight modules, such as the module $V(\mu)$ in Sect. 4.2, which was generated as a $U\left(s l_{n}\right)$-module by the vector $v_{0}$. It is well-known that a highest weight module for a quantized enveloping algebra is generated by $v_{0}$ as a $U^{-}\left(s l_{n}\right)$-module, where $U^{-}=U^{-}\left(s l_{n}\right)$ is the subalgebra of $U\left(s l_{n}\right)$ generated by the generators $f_{1}, \ldots, f_{n-1}$. For this reason it is helpful to describe the action of $f_{i}$ on the module $W(p)$. (We do not tackle the case of the action of the generators $e_{i}$ of $U^{+}\left(s l_{n}\right)$, but this can be understood with exactly parallel techniques.)

Definition 4.3.1. Let $\mu \in \Lambda(n, r)$. If $\mu_{i}>0$, we define $\mu^{-}(i)$ to be the element of $\lambda$ of $\Lambda(n, r)$ satisfying

$$
\begin{aligned}
\lambda_{i} & =\mu_{i}-1 \\
\lambda_{i+1} & =\mu_{i+1}+1 \\
\lambda_{k} & =\mu_{k} \text { otherwise. }
\end{aligned}
$$


Definition 4.3.2. Let $\lambda \in \Lambda(n, r)$ be such that $\lambda_{i} \leq 2$ for all $i$, and let $p$ be the number of $\lambda_{i}$ equal to 2 . Let $W_{\lambda}$ be the associated Weyl group. We define the generalised $(r, p)$ parenthesis diagram $\ell_{\lambda}$ to be that with label $\lambda$ and diagram $D$, where points $i$ and $j$ are joined by $a$ (flat) edge if and only if $j=i+1$ and $(i, i+1) \in W_{\lambda}$.

It was known ([1, Sect. 5.7], [4]) that the image of $f_{i}$ under the homomorphism $\pi_{r}$ from $U$ to $S_{q}(n, r)$ is given by

$$
\sum \theta_{\mu-(i), \mu}^{w\left(\mu^{-(i), \mu)}\right.}
$$

where $w(\lambda, \mu)$ is the longest element in the double coset $W_{\lambda} 1 W_{\mu}$ and the sum is taken over all $\mu \in \Lambda(n, r)$ for which $\mu^{-}(i)$ makes sense.

If we then project $\pi_{r}\left(f_{i}\right)$ to $Q(n, r)$ in the natural way and act it on the left on a diagram element of $W(p)$ (for some $p$ ) with label $\mu$, at most one term $\theta_{\mu^{-(i), \mu}}^{w\left(\mu^{-(i), \mu}\right.}$ will survive. (This follows from a simple argument based on the weight spaces of the $q$-Schur algebra.) Provided one term does survive, it is convenient to classify the situation as "case $1,2,3$ or 4" according to which of the conditions below is satisfied by $\mu$ :

$$
\begin{array}{ll}
\mu_{i}=2, & \mu_{i+1}=0, \\
\mu_{i}=2, & \mu_{i+1}=1, \\
\mu_{i}=1, & \mu_{i+1}=0, \\
\mu_{i}=1, & \mu_{i+1}=1 .
\end{array}
$$

If all the terms map to zero, we say we are in "case 0".

Definition 4.3.3. Suppose $i$ and $\mu$ are such that we are in case $1,2,3$ or 4 above. Let $D$ be the generalised $r$-diagram $\left|\ell_{\mu}\right\rangle\left\langle\ell_{\mu}\right|$ whose graph is $g$. Define the integers $a$ and $b$ by

$$
a:=1+\sum_{j=1}^{i-1} \mu_{j}
$$

and

$$
b:=\sum_{j=1}^{i+1} \mu_{j}
$$

This means that points $a, a+1, \ldots, b$ are precisely those points.in the diagram for $\ell_{\mu}$ with value $i$ or $i+1$. Note that no point in the vertical strip of $g$ containing points $a, a+1, \ldots, b$ in the top and bottom rows is connected to any point outside this strip.

We define a generalised $r$-diagram $f_{i}(\mu)$ with pair $\left\langle\mu^{-}(i), \mu\right\rangle$ by replacing the strip from point a to point $b$ (inclusive) in the graph $g$ in the following ways:

Case 1. Here $b=a+1$. We change the flat edge between points $a$ and $b$ in the top row of $D$ to a horizontal edge.

Case 2. Here $b=a+2$. Keep the flat edge joining points $a$ and $a+1$ in the bottom row where it is, but remove the other edges. Join points $a+1$ and $a+2$ in the top row with a flat edge, and connect point $a$ in the top row with point $a+2$ in the bottom row with a vertical edge.

Case 3. Here $b=a$. The graph $g$ remains unchanged; only the labels change.

Case 4. Here $b=a+1$. Remove the two vertical edges linking the points labelled $a$ and $b$. Join points $a$ and $b$ in the top row with $a$ flat edge, and points $a$ and $b$ in the bottom row with a horizontal edge. 
Proposition 4.3.4. Regard the set of generalised $(r, p)$-parenthesis diagrams as Lusztig's canonical basis for $V(\mu)$ as in Theorem 4.2.3. Then the left action of $f_{i}$ on a diagram d of $W(p)$ with label $\mu$ is given by the (usual) left action of $f_{i}(\mu)$ on $d$, if $f_{i}(\mu)$ is defined, and zero otherwise.

Proof. By projecting $f_{i}$ to $S_{q}(n, r)$ using $\pi_{r}$ and then to $Q(n, r)$ by taking the image after quotienting by $I$, one can easily check that Definition 4.3.3 makes this action compatible with the action of $f_{i}$ on Lusztig's canonical basis.

Remark. As was mentioned before, the case of the generators $e_{i}$ is very similar; essentially all that happens is that the diagrams produced in Definition 4.3.3 come out upside-down.

It is possible in theory to describe the action of the $f_{i}$ on the basis elements parametrised by tableaux instead of generalised $(r, p)$-parenthesis diagrams, but this is much less enlightening.

We conclude with an example in the case $n=5, r=5, p=3$.

Example 4.3.5. Let $\lambda=(2,2,1,0,0)$ and $\mu=(1,2,1,0,1)$. Consider the canonical basis element of $V(\lambda)$ given by diagram in Fig. 4 , or by the tableau

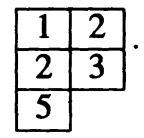

Then the action of $f_{2}$ falls into case 2 and is given by left multiplication by the element shown in Fig. 5, where $\mu^{-}(2)=(1,1,2,0,1)$.

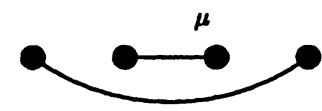

Fig. 4. Module basis element before the action of $f_{2}$

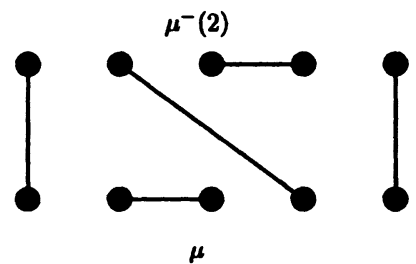

Fig. 5. Generalized $r$-Diagram corresponding to $f_{2}$

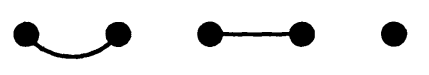

Fig. 6. Module element after the action of $f_{2}$ 
The result of the action is the basis element shown in Fig. 6, which corresponds to the tableau

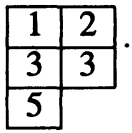

4.4. Remarks on the case $n<r$.. Finally we consider $Q(n, r)$, where $n<r$. Let us fix $N \geq r$. Note that there is a natural embedding $\iota$ of $\Lambda(n, r)$ into $\Lambda(N, r)$ which sends $\lambda \in \Lambda(n, r)$ to $\lambda^{\prime}$, where $\lambda_{i}^{\prime}=\lambda_{i}$ if $i \leq n$, and $\lambda_{i}^{\prime}=0$ otherwise.

Proposition 4.4.1. The algebra $Q(n, r)$ is canonically isomorphic to the subalgebra of $Q(N, r)$ spanned by basis elements $\zeta_{\lambda, \mu}^{x}$, where $\lambda$ and $\mu$ are of the form $\iota\left(\lambda_{1}\right)$ and $\iota\left(\mu_{1}\right)$ respectively, for some $\lambda_{1}, \mu_{1} \in \Lambda(n, r)$.

Proof. This is immediate from the fact that the $\mathcal{A}$-linear map sending $\theta_{\lambda, \mu}^{w^{+}} \mapsto \theta_{\iota(\lambda), \iota(\mu)}^{w^{+}}$ is an algebra monomorphism, which is an easy consequence of the definition of the $\theta$ basis.

Acknowledgement. The author is grateful to C.K. Fan for some helpful discussions, and also to K. Erdmann and R.J. Marsh for many useful comments on the manuscript.

\section{References}

1. Beilinson, A.A., Lusztig, G. MacPherson, R.: A geometric setting for the quantum deformation of $G L_{n}$. Duke. Math. J. 61, 655-677 (1990)

2. Dipper, R. James, G.D.: The $q$-Schur algebra. Proc. L.M.S. 59, 23-50 (1989)

3. Du,J., Canonical Bases for Irreducible Representations of Quantum $G L_{n}$. Bull. London Math. Soc. 24, 325-334 (1992)

4. Du, J.: A Note on Quantized Weyl Reciprocity at Roots of Unity. Algebra Colloq. 4, 363-372 (1995)

5. Du, J.: Canonical Bases for Irreducible Representations of Quantum $G L_{n}, I I$. J. Lond. Math. Soc. (2) 51, 461-470 (1995)

6. Fan, C.K., Green, R.M.: Monomials and Temperley-Lieb Algebras. J. Alg., to appear

7. Graham, J.J., Lehrer, G.I.: Cellular Algebras. Invent. Math. 123, 1-34 (1996)

8. Green, R.M.: Positivity Properties for $q$-Schur Algebras. Proc. Cam. Phil. Soc., to appear

9. Lusztig, G.:Canonical Bases Arising from Quantized Enveloping Algebras. J. Am. Math. Soc. 3, 447498(1990)

10. Shi Jian-yi: The Kazhdan-Lusztig cells in the affine Weyl group. Lecture Notes in Mathematics, 1179, New York: Springer, 1986

11. Temperley, H.N.V., Lieb, E.H.:Relations between percolation and colouring problems and other graph theoretical problems associated with regular planar lattices: some exact results for the percolation problem. Proc. Roy. Soc. Lond. Ser. A 322, 251-280 (1971)

12. Westbury, B.W.:The representation theory of the Temperley-Lieb Algebras. Math. Z. 219, 539-565 (1995) 